\title{
On the global flow-field dynamics around an airfoil
}

\author{
Václav Uruba ${ }^{1,2, *}$, Pavel Procházka ${ }^{1}$, and Vladislav Skála ${ }^{1}$ \\ ${ }^{1}$ Institute of Thermomechanics, ASCR, v.v.i., Dolejškova 5, Praha 8, CR \\ ${ }^{2} \mathrm{UWB}$, FME, Department of Power System Engineering, Universitní 8, Plzeň, CR
}

\begin{abstract}
The global flow-field around an airfoil will be studied using time-resolved PIV technique. Interactions between pressure and suction sides flows will be studied in detail. Spanwise structures dynamics is to be analysed.
\end{abstract}

\section{Introduction}

The flow around an airfoil belongs to so-called canonical cases. It has been studied for very long period using all available methods - experimental and mathematical see e.g. [4]. Prevailing amount of results deal with averaged quantities - velocity, vorticity, pressure fields. Despite all that, the details are not still clear, as the flowfield in the boundary layers and in the wake is highly unsteady and 3D.

On the unexplored 3D instantaneous structure of the airfoil boundary layer on its suction side the new theory of flight by Hoffman and Johnson from KTH Stockholm is based, see $[1,2]$. The hypothesis of physical mechanism of flight relies on existence of streamwise vortical structures on the suction side of the airfoil and within its wake. The vortices origin is supposed to be the instability of the boundary layer subjected to adverse pressure gradient on the airfoil suction side (i.e. upper) see [3].

Our research group published recently several experimental studies on 3D instantaneous structure of the flow around a simple airfoil, e.g. $[5,6,9,10,11]$. The presented study is oriented on the existence of spanwise-oriented structures and their dynamics and merging with each other along the profile chord. As in the previous experiments, the simplest airfoil represented by an inclined flat plate is used in this paper. The plate chord $c$ is the reference length.

In our preceding studies the presence of streamwise vorticity within the turbulent boundary layer on the suction side of simple airfoil was proved experimentally - see e.g. [10]. Within the boundary layer the vorticity is typically distributed randomly both in time and space (spanwise position).

The boundary layer thickness was detected about $0.2 \mathrm{c}$ in the trailing edge position. The acquired data clearly revealed the presence of the streamwise vortices of both orientations within the airfoil suction side boundary layer. The vortices are variable both in sizes and locations. To recognize typical vortex parameters, further analysis using POD method was performed. It has revealed appearance of similar vortices quasiperiodically in span direction. Several high-energy POD modes suggested existence of contra-rotating vortex pairs oriented in streamwise direction. More details on the POD analysis of velocity fields in planes perpendicular to the flow could be find in [10] and in [7, $8]$ on the POD method itself.

To complete information about the spanwise-oriented vortices and their dynamics we prepared the new persuasive experiment. The TR-PIV measurement conducted for longitudinal plane of measurement (see figure 1) perpendicular to the inclined plate was carried out. The task of this research is to investigate the separation bubble behind the leading edge and also its dynamic activity. The wake region behind the trailing edge was explored as well as the whole region around an airfoil. The overall view is important to study mutual influence of the pressure and suction sides flows. To fulfil the defined objectives, the statistical quantities (mean values, statistical central moments etc.) and mainly POD (Proper Orthogonal Decomposition) is utilized.

\section{Experimental setup}

The experiments were carried out in the blow-down facility in IT.

\subsection{Model}

Flat plate inclined with angle of attack (AOA) 7 degrees has been placed in a uniform low turbulent stream. The blow-down wind tunnel produces a jet with uniform velocity distribution, mean velocity about $5 \mathrm{~m} / \mathrm{s}$, intensity of turbulence less than $0.2 \%$. The plate of thickness $2 \mathrm{~mm}$ has rounded edges, chord is $100 \mathrm{~mm}$ and span $450 \mathrm{~mm}$. The Reynolds number based on plate chord is about 33 thousands. The scheme of the experiment is in figure 1 .

The plane of measurement is located parallel to the channel axis and perpendicular to the flat plate surface.

\footnotetext{
* Corresponding author: uruba@it.cas.cz
} 
The Cartesian coordinate system is introduced with $\mathrm{x}$ axis in streamwise direction parallel to the plate surface, $z$ in spanwise direction and $y$ perpendicular to the plate surface. The origin of the coordinates is fixed at the centre of the leading edge (red coordinates in figure 1).

The velocity distributions and all POD modes will be presented for plane of measurement (green in figure 1) which was established in the centre of the airfoil.

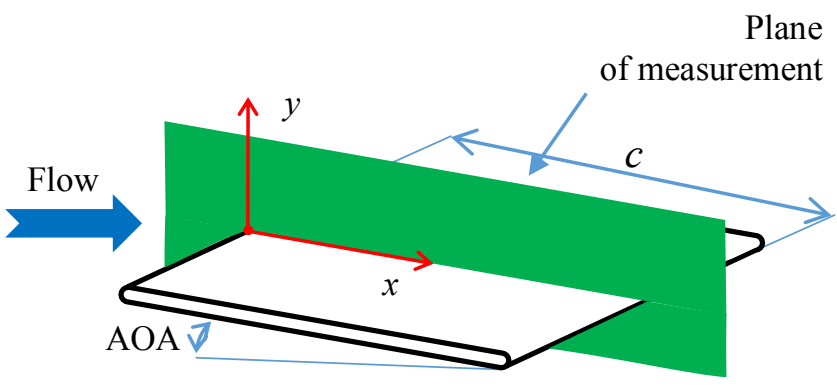

Fig. 1. Schematics of the experiment.

\subsection{Instrumentation}

The time-resolved PIV measuring system was used for the experiments.

The measuring system DANTEC consists of laser with cylindrical optics and one CCD camera with lens $60 \mathrm{~mm}$ focal length. Laser New Wave Pegasus Nd:YLF, double head, wavelength $527 \mathrm{~nm}$, maximal frequency $10 \mathrm{kHz}$, a shot energy is $10 \mathrm{~mJ}$ for $1 \mathrm{kHz}$ (corresponding power $10 \mathrm{~W}$ per head). The camera Phantom V611, resolution $1280 \times 800$ pixels and frequency up to 3000 double-snaps per second, memory is $8 \mathrm{~GB}$. Tracing particles with mean diameter value of $1 \mu \mathrm{m}$ were introduced to the flow upstream the test section. Software Dynamic Studio ver. 3.4 was used to data acquisition and post-processing.

The 1000 double-snaps were acquired in frequency $100 \mathrm{~Hz}$ for each experiment, to obtain good statistics in $10 \mathrm{~s}$ of the record time. To study the dynamics of the flow, the acquisition frequency was set to $2 \mathrm{kHz}$, records of $2 \mathrm{~s}$ were acquired.

\subsection{Analysis methods}

The vector evaluation was conducted using "adaptive correlation" algorithm resulting in velocity vector maps with resolution $157 \times 97$ vectors. The dimensions of flow domain were varying and can be seen from presented figures.

The main part of this article is an effort to extract energetic dominant structures present in a complex fully turbulent flow using decomposition (POD) method. This analysis is performed from fluctuation, see [7, 8]. The POD modes are ordered according to the relative kinetics energy content, which means that first modes are the most important, containing the biggest structures with high amplitudes. The number of resulting orthogonal modes is equal to number of all PIV snapshots. For precise POD analysis, it is necessary to crop the field of view because the analysis is very sensitive to invalid vectors (which are present e.g. in front of the airfoil due to laser shadow) and also it is suitable to study each flow phenomenon independently.

\section{Results}

The results are to be presented in specific spatial domains, first for whole airfoil, then for the leading edge and wake regions respectively. Last paragraph will present the dynamical analysis.

\subsection{Whole airfoil}

The principal results on mean flow around the whole airfoil are in figure 2.
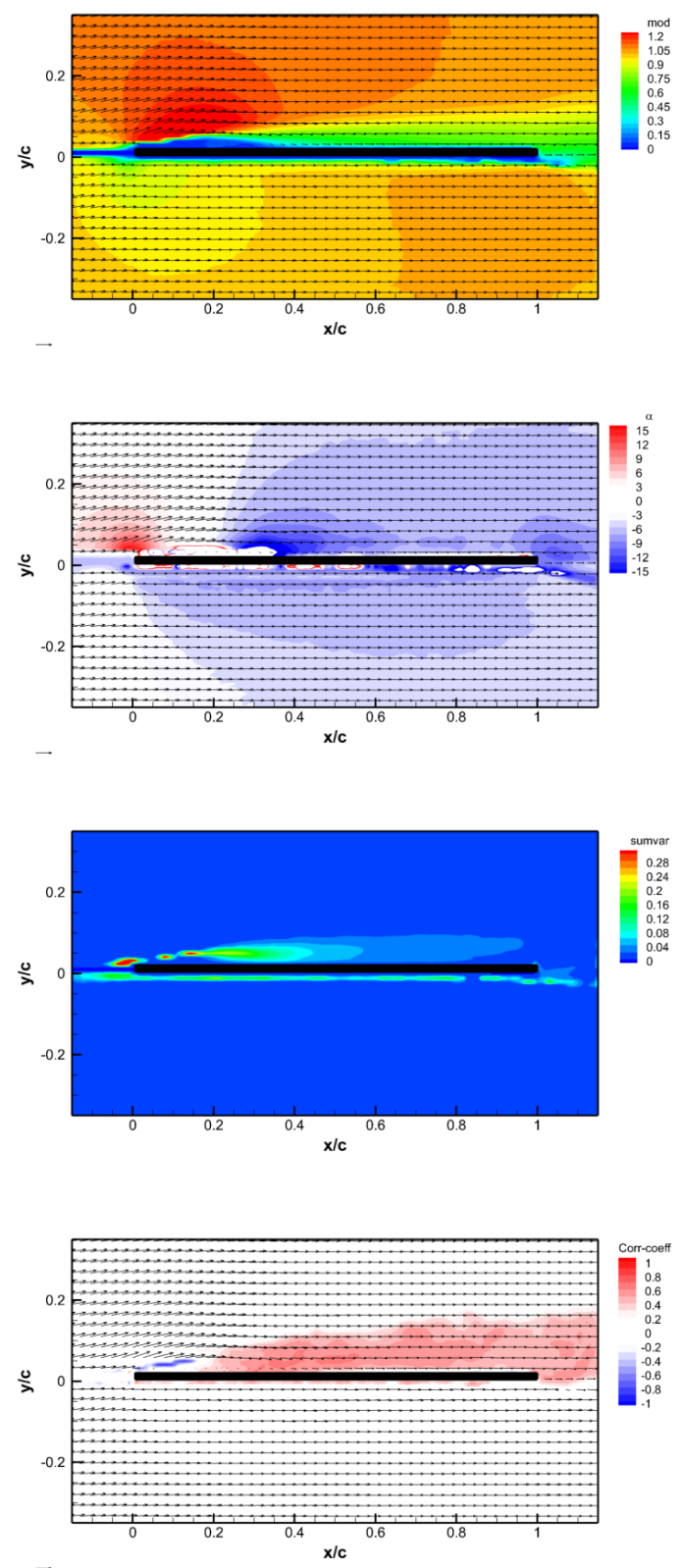

Fig. 2. Mean velocity, down-wash angle, TKE and correlation coefficient distributions, whole airfoil. 
The mean velocity modulus (mod), down-wash angle $(\alpha)$, turbulent kinetic energy (TKE, sumvar) and correlation coefficient distributions are presented respectively.

Firstly, the topology of global flow-field around flat plate is presented. Note, that the coordinate system is aligned with the plate's surface. Hence, the incoming freestream is oriented from left to right side from below $\left(\right.$ AoA $\left.=7^{\circ}\right)$. The vector in the left-hand lower corner corresponds to the dimensionless velocity 1 (the velocity is equal to velocity of incoming flow).

Separation bubble is created immediately behind the leading edge and its extent is up to $x / c=0.3$. The point of re-attachment is not steady in time. The overspeed region is apparent above the separation (velocities are about $20 \%$ higher than freestream). The boundary layer thickness is significantly bigger for the suction (upper) side, this could be related to existence of vortical structures. The flow velocity is decreased below the leading edge and consecutively it is accelerated toward the trailing edge to value of freestream velocity.

Second distribution on figure 2 is devoted to the down-wash angle. White colour means zero deviation from AoA ( $7^{\circ}$ considering to airfoil), while light blue colour means deviation equal to $-7^{\circ}$, i.e. vectors are oriented parallel to the surface. This feature is characteristic for whole pressure side region. The biggest positive deviation (red colour) is above the separation bubble at the leading edge. On the other hand, the biggest negative deviation is right behind the separation zone as the velocity vectors are oriented toward the airfoil surface.

The distribution of TKE indicates the fluctuation activity around the flat plate. The highest value is present at the boundary of the separation bubble. This means that the size and position of the separation is not steady. Further downstream, the fluctuation activity is significant for entire suction side. On contrary, there are almost no fluctuations in pressure lower side.

The correlation coefficient distribution reveals the areas where turbulence production process take place. The positive value can be seen in the whole area of suction side behind the separation. There is nearly no production in lower side.

\subsection{Leading edge region}

The close-up on leading edge region gives more detailed information that is in a good agreement with previous results for the whole region. The upstream half of the flat plate is observed together with some space upstream the leading edge. It is necessary to mention that information about flow upstream the leading edge is incomplete because the laser sheet illumination was performed from behind and there is a shadow (compare the blue region upstream the leading edge in the first figure 3).

The flow is separated as laminar boundary layer. Then the transition to turbulence takes place within a free shear layer and the flow is re-attaches creating the turbulent boundary layer.
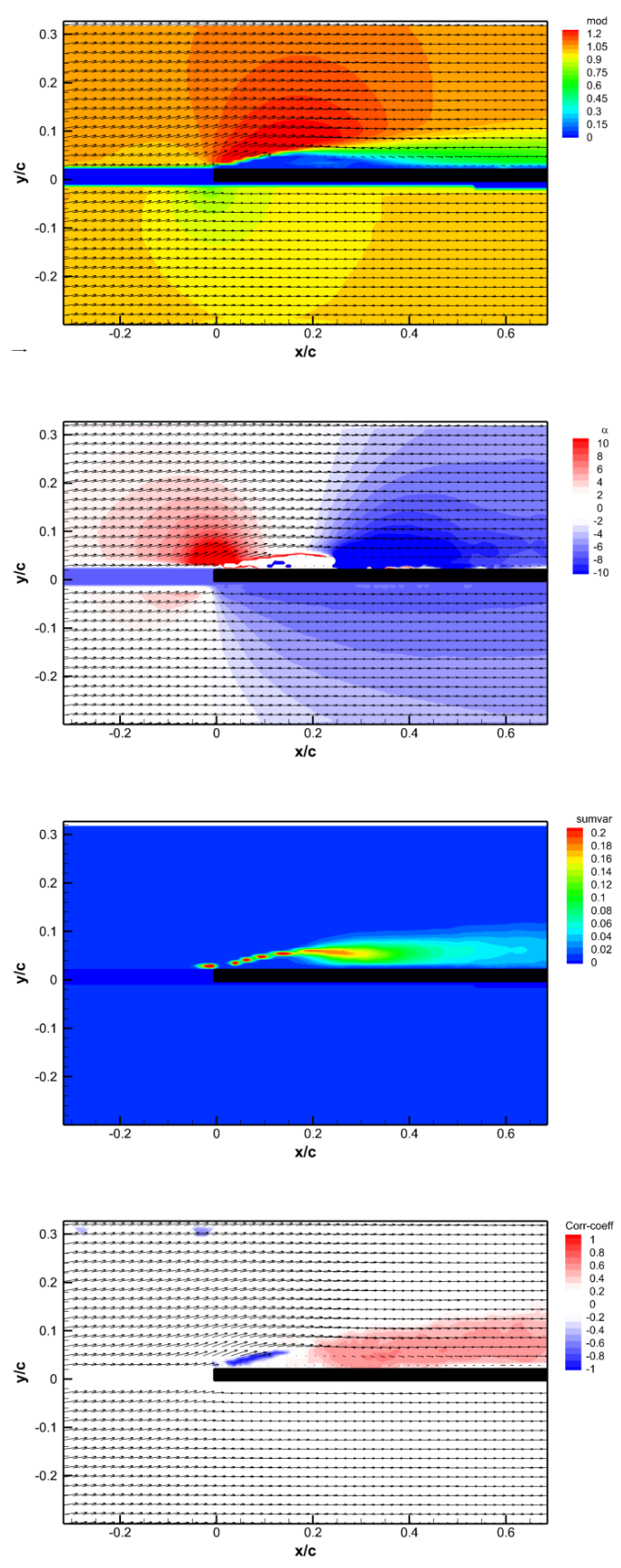

Fig. 3. Mean velocity, down-wash angle, TKE and correlation coefficient distributions, the area of separation bubble.

\subsection{Wake region}

Very interesting results were obtained within the wake region. Figure 4 contains again the distributions of four statistical quantities as in previous cases. The trailing edge is located at dimensionless coordinate $x / c=1$. 

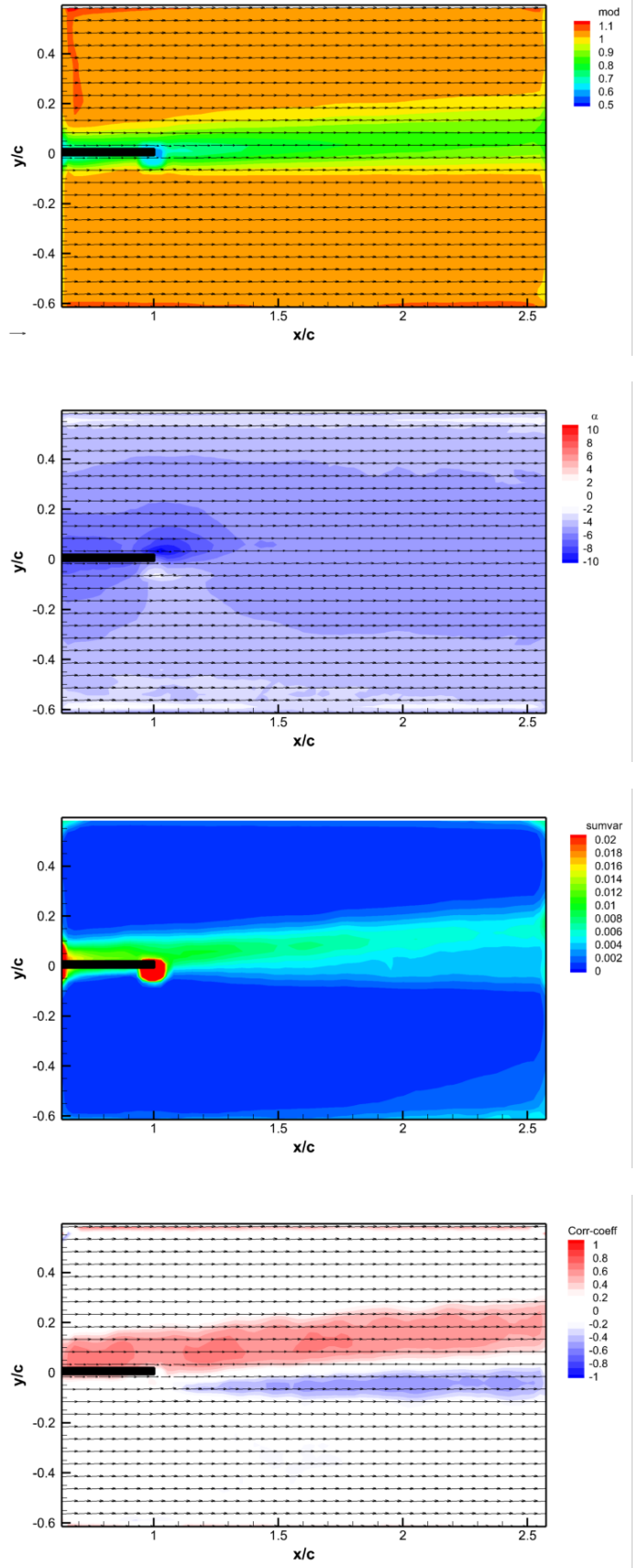

Fig. 4. Mean velocity, down wash angle, TKE and correlation coefficient distributions, the wake.

The wake is characterised by decreased velocities because of boundary layers. The wake width slightly increases with streamwise coordinate. There is a von Karman vortex street (are not visible from mean flow field) in the wake. The vortex shedding frequency is approximately $300 \mathrm{~Hz}$ and $1000 \mathrm{~Hz}$ for incoming velocity $5 \mathrm{~m} / \mathrm{s}$ and $10 \mathrm{~m} / \mathrm{s}$, respectively. Generally it can be said that the wake is full of spanwise as well as streamwise oriented vortical structures. The fluctuation activity is apparent mainly at suction side of the plate and further in the wake. The correlation coefficient is positive in the upper half of the wake, while lower part is characterised by a negative value.

\section{Flow dynamics}

The POD decomposition is suitable tool to detect most important vortex structures from turbulent flow field. Although the highest-order modes represent very often only noise, all 4000 POD modes were evaluated.

First, we focused on the region of separation bubble and slightly further downstream. The figure 5 shows POD modes Nos. 1, 3, 5 and 7. The black rectangle represents the airfoil location.
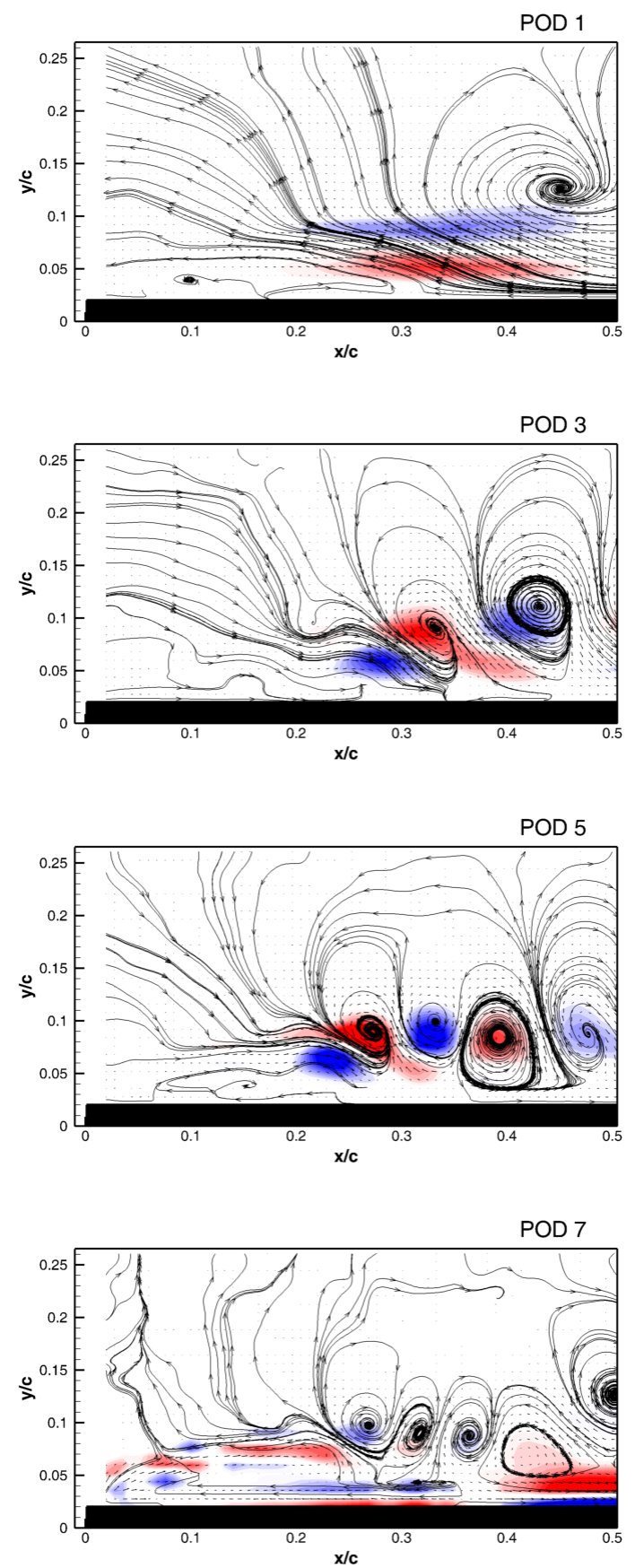

Fig. 5. Some examples of POD evaluated for first half of the suction side.

The streamlines are used to make individual vortical structures visible. The distribution of vorticity (redpositive, blue-negative) is applied. The region of 
separation is quite good visible from all 4 modes. The remarkable vortices can be detected behind $1 / 3$ of the chord length in the boundary layer. The first POD mode contains one dominant vortical structure, while other higher modes contain a pair of counter-rotating vortices or even more. It is obvious that spanwise vorticity is present in the turbulent boundary layer.

Figure 6 is a representation of relative kinetic energy content of the first 10 POD modes evaluated at separation region.

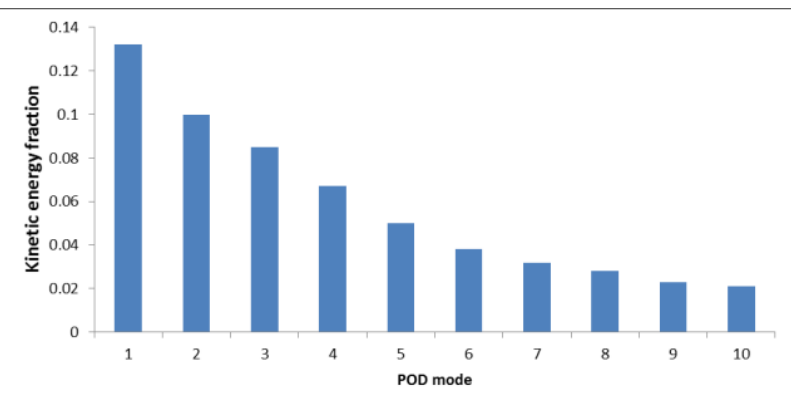

Fig. 6. Relative kinetic energy content of the first 10 POD modes, the leading edge.

The last group of results presents the significant POD modes evaluated inside the wake. Note that the second and third mode did not contain any vortical structure in the wake despite the fact that both contain approx. $10 \%$ of kinetic energy. Both modes are characterized by weak shear layers behind the trailing edge. Furthermore, the third mode is characterized by existence of a vortex at upper side right upstream the trailing edge. The direction of the streamlines is not relevant as the POD modes represent fluctuation components.

The modes number 6,9 and 10 are depicted here to show the existence of spanwise vorticity above the airfoil and mainly in the wake region. One can observe miscellaneous vortices. Lower-order modes contain bigger structures with more significant energy content, higher order modes contain higher number of structures of smaller diameter.

Figure 8 is plotted to show the relative energy content of 10 low-order modes calculated in the wake region. The energies of the presented modes are between 2 and $4 \%$.

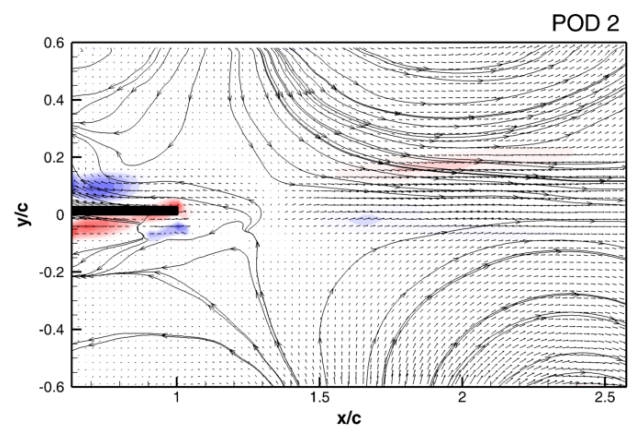

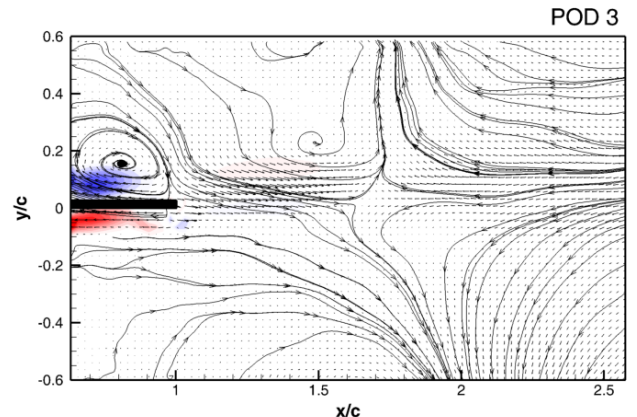
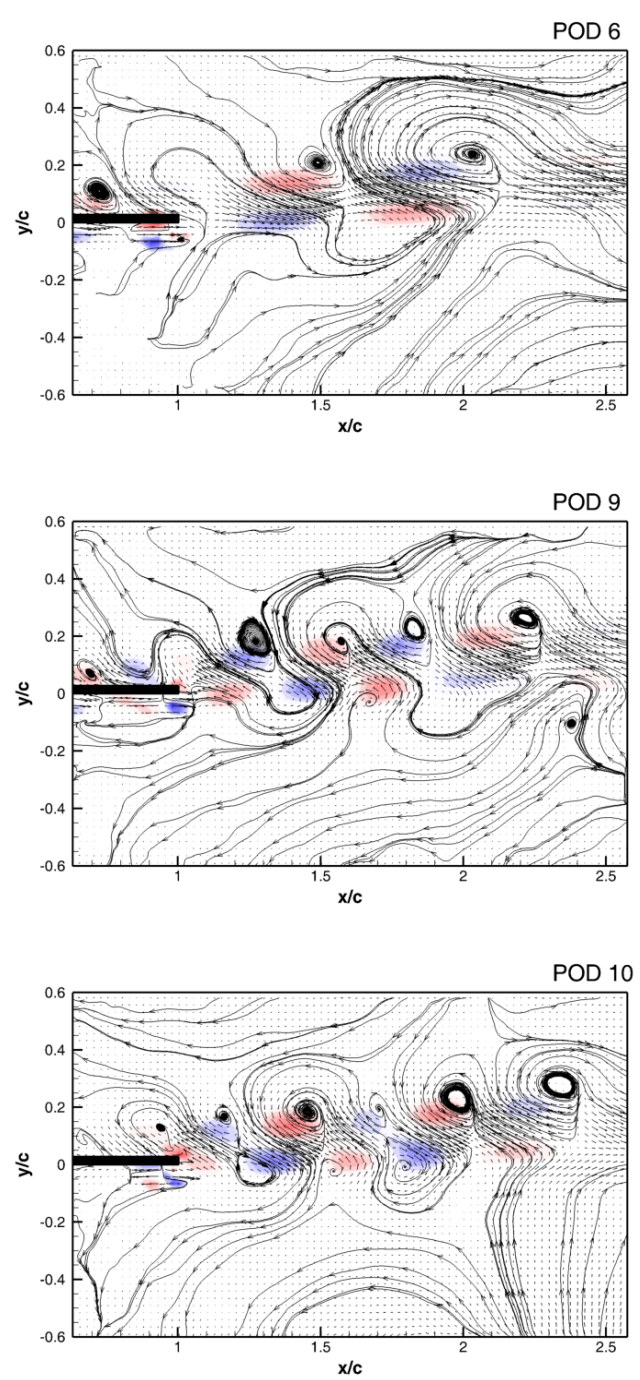

Fig. 7. Some examples of POD evaluated for the wake behind the flat plate. 


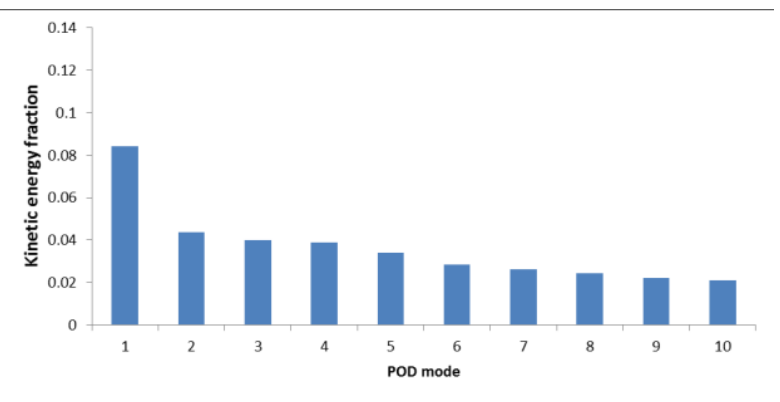

Fig. 8. Relative kinetic energy content of first ten POD modes, the wake region.

The energy of the first mode (not shown here) is over $8 \%$. The scale of the kinetic energy fraction is same in figures 6 and 8 for better comparison.

\section{Conclusions}

The experimental investigation of spanwise vortices was performed using TR-PIV measurement technique. The data were presented to demonstrate the topology of the flow field, especially separation bubble, and to show the area of maximal fluctuation activity. Furthermore, the down wash angle value along the flat plate was investigated. Dynamics of the flow was examined using POD decomposition.

It is clear that particularly flow-field within the suction side boundary layer of the airfoil is full of spanwise and streamwise vorticity. Rotational slip separation condition, which was suggested to supply new theory of flight and lift $[1,2]$, is much more complex physical phenomenon and additional and more elaborate procedures should be applied.

Next studies are required to describe the phenomenon properly.

This work was supported by the Grant Agency of the Czech Republic, project No. 17-01088S.

\section{References}

1. J. Hoffman, C. Johnson, The Mathematical Secret of Flight, Normat 57, 4, pp.1-25, (2009)

2. J. Hoffman, C. Johnson, Resolution of d'Alembert's paradox, J. Math. Fluid Mech. 12 (3), pp.321-334, (2010)

3. P. Manneville, Instabilities, Chaos and Turbulence. Imperial College Press, 2004

4. P. Schlatter, Q. Li, R. Örlü, F. Hussain, D.S. Henningson, On the near-wall vortical structures at moderate Reynolds numbers, European Journal of Mechanics B/Fluids 48 (2014) 75-93

5. P. Procházka, V. Uruba, V. Skála, On the 3D structure of the flow-field in the vicinity of inclined plate, J. of Phys.: Conference Series publication (to be published)
6. P. Procházka, V. Uruba, V. Skála, Evolution of vortical structures behind an inclined flat plate, MATEC Web of Conferences 168 (2018) Article number 05003

7. V. Uruba, Near Wake Dynamics around a Vibrating Airfoil by Means of PIV and Oscillation Pattern Decomposition at Reynolds Number of 65000 , Journal of Fluids and Structures, 55, pp. 372-383 (2015)

8. V. Uruba, Decomposition methods in turbulent research, EFM11, EPJ Web of Conferences, 25 01095 (2012)

9. V. Uruba, P. Procházka, V. Skála, On 3D Flow Structure of the Boundary Layer on the Suction Side of a Plate, EPJ Web of Conferences 180 (2018) Article number 02112

10. V. Uruba, D. Pavlik, P. Prochazka, V. Skala, V. Kopecky, On 3D flow-structures behind an inclined plate, EPJ Web of Conferences 143 (2017) Article Number UNSP 02137

11. V. Uruba, Z. Pátek, P. Procházka, V. Skála, D. Zacho, R. Kulhánek, Flow Structure behind a Wing at High Reynolds Numbers, EPJ Web of Conferences 180 (2018) Article number 02111 